CERN-TH/2003-127

SLAC-PUB-9929

ULB-TH-03/22

ITFA-2003-30

hep-th/0306127

\title{
Comments on the Gauge Fixed BRST Cohomology and the Quantum Noether Method ${ }^{1}$
}

\author{
Glenn Barnich ${ }^{2}$ \\ Physique Théorique et Mathématique, Université Libre de Bruxelles \\ Campus Plaine C.P. 231, B-1050 Brussels, Belgium \\ gbarnich@ulb.ac.be \\ Tobias Hurth ${ }^{3}$ \\ CERN, Theory Division, CH-1211 Geneva 23, Switzerland \\ SLAC, Stanford University, Stanford, CA 94309, USA \\ tobias.hurth@cern.ch \\ Kostas Skenderis \\ Institute for Theoretical Physics, University of Amsterdam \\ Valkenierstraat 65, 1018 XE Amsterdam, The Netherlands \\ skenderi@science.uva.nl
}

\begin{abstract}
We discuss in detail the relation between the gauge fixed and gauge invariant BRST cohomology. We showed previously that in certain gauges some cohomology classes of the gauge-fixed BRST differential do not correspond to gauge invariant observables. We now show that in addition "accidental" conserved currents may appear. These correspond one-toone to observables that become trivial in this gauge. We explicitly show how the gauge-fixed BRST cohomology appears in the context of the Quantum Noether Method.
\end{abstract}

\footnotetext{
${ }^{1}$ Based on an invited lecture presented by T.H. at the Hesselberg Meeting 2002 "Theory of Renormalization and Regularization", Hesselberg, Germany; a short version of the paper without background material will appear in Physics Letters B

${ }^{2}$ Research Associate of the National Fund for Scientific Research (Belgium)

${ }^{3}$ Heisenberg Fellow
} 


\section{Introduction}

Noether's theorem in classical field theory allows for an iterative construction of invariant classical actions [1]. Starting from a given Lagrangian that is invariant under some symmetry, one can iteratively construct interactions by adding extra terms to the action and to the transformation rules, so that the final action is invariant. In the simplest case the starting Lagrangian is free. In [2, 3] a construction of theories with global (=rigid) and/or local symmetries within the Bogoliubov-Shirkov-Epstein-Glaser (BSEG) approach was presented. This construction may be viewed as a quantum version of the classical Noether method.

In the BSEG approach, the $S$-matrix is directly constructed in the Fock space of free asymptotic fields as a formal power series. The coupling constant is replaced by a tempered test function $g(x)$ (i.e. a smooth function rapidly decreasing at infinity) which switches on the interaction. Instead of obtaining the $S$-matrix by first computing off-shell Green functions by means of Feynman diagrams and then applying the LSZ formalism, the $S$-matrix is obtained by imposing causality and Poincaré invariance. The method can be regarded as an "inverse" of the cutting rules: one builds $n$-point functions out of $m$-point functions $(m<n)$ by suitably "gluing" them together. The precise manner in which this is done is dictated by causality and Poincaré invariance. It can be shown that this process uniquely fixes the $S$-matrix up to local terms (for a brief introduction to the BSEG method see section 3 of [2]; for more detailed accounts the reader is referred to 4, 5, 6, 7, 8, ).

The BSEG approach should not be regarded as a special renormalization scheme but as a general framework in which the conditions posed by the fundamental axioms of quantum field theory (QFT) on any renormalization scheme are built in by construction. In this sense the quantum Noether construction in [2, 3] is independent from the causal BSEG approach. The proposed quantum Noether conditions should hold in any other formalism.

In the Lagrangian framework, powerful methods have been developed that allow for a systematic cohomological analysis of local counterterms and anomalies [9]. The BRSTBV method provides an efficient way of analysing Ward identities. In the BSEG operator approach, Ward identities play a central role: they provide the quantum Noether conditions (QNC) 2, 3]. Consistency then requires that in the (naive) adiabatic limit, $g(x) \rightarrow 1$, the constraints imposed by the QNC be equivalent to those obtained via the antifield formalism. This issue was analysed in [10]. We will review and further elaborate on these results in this contribution. In particular, we analyze in detail the issue of the coboundary condition which we only touched upon in [10]. 
The paper is organized as follows. In sections 2 and 3 the antifield formalism is discussed. Section 4 is devoted to the discussion of the relation between the gauge fixed and gauge invariant BRST cohomology. In section 5 we present an example that illustrates aspects of the analysis in section 4 . In section 6 we recall the quantum Noether method and in section 7 we use the results of section 4 to analyse the general solution of the quantum Noether conditions. The appendix contains a brief review of the off-shell procedure used in BSEG computations.

\section{Renormalization with Antifields}

The problem of perturbative quantization of generic gauge theories in the Lagrangian framework consists of renormalizing the theory while preserving an appropriate form of gauge invariance at the quantum level. We briefly summarize the standard approach here (we refer to [11, 12, 13, for reviews).

The first step is the introduction of an appropriate number of ghost fields. More precisely, independent ghost fields are associated to each element of the generating set of gauge transformations of the theory, ghosts-for-ghosts for each element of the generating set of reducibility operators, additional ghosts for the reducibility operators of the reducibility operators, and so on. The collection of original gauge and matter fields and of all the ghosts will be called fields and denoted $\phi^{A}$ in the following.

For each field $\phi^{A}$, one introduces an antifield $\phi_{A}^{*}$, which allows for a definition of an odd graded Lie bracket, the antibracket, in the space of functionals of fields and antifields ${ }^{4}$,

$$
(\cdot, \cdot)=\frac{\delta^{R} \cdot}{\delta \phi^{A}} \frac{\delta^{L} \cdot}{\delta \phi_{A}^{*}}-\frac{\delta^{R} \cdot}{\delta \phi_{A}^{*}} \cdot \frac{\delta^{L}}{\delta \phi^{A}}
$$

where right and left derivatives are defined by $\delta F=\left(\delta^{R} F / \delta z^{\alpha}\right) \delta z^{a}=\delta z^{a}\left(\delta^{L} F / \delta z^{\alpha}\right)$.

Using the antifields, one couples to the original gauge invariant action the gauge transformations with gauge parameters replaced by ghosts, and also the various reducibility operators with their parameters replaced by ghosts-for-ghosts. This action can then be completed to the so-called solution of the master equation $S\left[\phi, \phi^{*}\right]$ at ghost number 0 satisfying

$$
(S, S)=0 .
$$

The associated BRST differential $s$ is canonically generated in the antibracket by the solution of the master equation, $s=(S, \cdot)$; it acts in particular on both the fields and the antifields.

\footnotetext{
${ }^{4}$ We use DeWitt's condensed notation.
} 
For gauge-fixing purposes, a non-minimal sector is added to $S$. Usually, it consists of adding auxiliary fields coupled to the antifields of the antighosts. Then one chooses a gauge-fixing fermion $\Psi$ that depends only on the fields of the original and the nonminimal sector. After performing a canonical transformation generated by $\Psi$, the solution of the master equation has an invertible kinetic part for the fields and can be used as a starting point for perturbative quantization. Formal path integral arguments then show the independence of correlation functions of BRST invariant operators on the choice of the gauge-fixing fermion. One of the main problems is to investigate to what extent the same property holds true in the renormalized theory.

In the power-counting renormalizable case, general results imply that the quantum counterpart of (2.2) can be expressed in terms of the generating functional $W\left[j, \phi^{*}\right]$ of connected Green functions in the presence of antifields ${ }^{5}$ as the anomalously broken Slavnov-Taylor identity

$$
j_{A} \frac{\delta^{L} W}{\delta \phi_{A}^{*}}=\hbar\langle\mathcal{A}\rangle,
$$

with $\mathcal{A}$ a local integrated functional of ghost number 1. Equivalently, when expressed in terms of the generating functional $\Gamma\left[\phi, \phi^{*}\right]$ of 1PI Green functions, the anomalous Slavnov-Taylor identity (2.3) becomes the anomalous Zinn-Justin equation

$$
\frac{1}{2}(\Gamma, \Gamma)=\hbar \mathcal{A} \circ \Gamma
$$

The appropriate form of gauge invariance that a renormalizable theory should satisfy at the quantum level is the non-anomalous Slavnov-Taylor identity, or equivalently the non-anomalous Zinn-Justin equation.

The anomalous breaking $\mathcal{A} \circ \Gamma$ satisfies the consistency condition

$$
(\Gamma, \mathcal{A} \circ \Gamma)=0
$$

which implies to lowest non-trivial order in $\hbar$ that the anomaly $\mathcal{A}$ satisfies the consistency condition $s \mathcal{A}=0$. Because BRST exact anomalies can be absorbed by BRST breaking counterterms at ghost number 0 , a sufficient condition for the absence of the lowest order anomaly is the vanishing of the BRST cohomology $H^{1}(s)$ in the space of local functionals of fields and antifields at ghost number 1 .

Suppose now that there are no non trivial anomalies and that, to lowest order, the trivial anomalies have been canceled by BRST breaking counterterms. If one wants

\footnotetext{
${ }^{5}$ In the case of Yang-Mills gauge theories, the antifields that appear in the gauge fixed action correspond to sources for the BRST variations of the fields. From the point of view of renormalization, they are needed because not only the terms in the action, but also the non-linear BRST transformations, are subject to quantum corrections.
} 
to preserve the non-anomalous Zinn-Justin equation to that order, the remaining finite counterterms $C$ must be BRST invariant, $s C=0$. (In a regularization-renormalization approach, it follows from (2.4) that the lowest order divergences $D$, which are integrated local functionals in ghost number 0 , are also required to be BRST invariant, $s D=0$ ). BRST exact counterterms can be absorbed by canonical field-antifield redefinitions. It can then be shown that a sufficient condition that allows for an iterative proof of the renormalizability of the theory, while preserving the non-anomalous Zinn-Justin equation, is the property of stability, namely that, to each element of the BRST cohomology $H^{0}(s)$ in the space of local functionals of fields and antifields at ghost number 0 , there corresponds an independent coupling constant in the action.

Note that from the point of view of Green functions, the antifields are merely an (extremely efficient) bookkeeping device, which tell how certain combinations of Green functions with certain insertions of operators should be renormalized.

\section{Cohomological Considerations}

In this section, we discuss local BRST cohomology with antifields included (see [14 for a review and references).

In the renormalization of gauge theories, an important part is played by local BRST cohomology or, more precisely, by the cohomology groups of the BRST differential $s$ at ghost number 0 and 1 in the space $\mathcal{F}$ of local functionals of the fields and the antifields. Even though the BRST differential that arises directly in the problem of renormalization is the one associated to the non-minimal and gauge fixed solution of the master equation $S$, the crucial point is that the cohomology is isomorphic to the canonical, gauge invariant BRST cohomology of the minimal, non-gauge fixed solution of the master equation.

The information contained in the BRST cohomology groups involving the antifields can be described entirely without these antifields. Indeed, consider an expansion of the BRST differential in canonical form according to the canonical antifield number, which consists of assigning degree 0 to the fields, degree 1 to the antifields of the original gauge and matter fields, degree 2 to the antifields of the ghosts, degree 3 for those of the ghosts for ghosts, etc., so that $s=\delta+\gamma+s_{1}+\ldots$ Here $\delta$ lowers the antifield number by 1 and is related to the original gauge invariant equations of motion. The operator $\gamma$ is of degree 0 and its action on the gauge and matter fields, after putting the antifields to zero, corresponds to gauge transformations with gauge parameters replaced by ghosts, while its action on the fields of the non-minimal sector is trivial. Finally, the operators $s_{k}$ are of degree $k$. It turns out that at all positive ghost numbers $g$, the BRST cohomology in $\mathcal{F}$ is isomorphic to the cohomology of the differential $\gamma$ in the space of local on-shell 
functionals $\mathcal{F}_{W}$ depending on the fields alone:

$$
H^{g}(s, \mathcal{F}) \simeq H^{g}\left(\gamma, \mathcal{F}_{W}\right), g \geq 0
$$

By on-shell functionals, we mean equivalence classes of functionals modulo functionals that vanish when the gauge invariant equations of motion of the original theory hold.

Furthermore, at ghost number -1 , the BRST cohomology in $\mathcal{F}$ is isomorphic to the space of equivalence classes of global symmetries of the original gauge invariant theory, where two global symmetries are equivalent if they differ on-shell by a gauge symmetry. This last space turns out to be isomorphic to the space of equivalence classes of conserved currents modulo divergences of superpotentials and on-shell vanishing currents. For irreducible gauge theories, at ghost number -2 , the BRST cohomology in $\mathcal{F}$ is isomorphic to the space of equivalence classes of global reducibility identities, i.e. to classes of field dependent parameters of gauge transformations that make the corresponding gauge symmetries vanish on-shell, modulo on-shell vanishing gauge parameters. This space is, in turn, isomorphic to the space of equivalence classes of conserved superpotentials modulo divergences of skew-symmetric rank 3 contravariant tensors (depending locally on the original gauge and matter fields and their derivatives) and on-shell vanishing superpotentials. The BRST cohomology (for irreducible gauge theories) in $\mathcal{F}$ in ghost number less than -2 vanishes.

Because it is the gauge fixed form of the BRST symmetry that arises naturally in the problem of renormalization, it is sometimes useful to describe the content of the local BRST cohomology groups in terms of the gauge fixed theory, without the associated antifields. In the gauge fixed form, the appropriate expansion of the differential $s$ is according to the gauge fixed antifield number, which consists of assigning antifield number 0 to the fields and antifield number 1 to all the antifields, so that $\tilde{s}=\delta^{g}+\gamma^{g}+\lambda^{g}+\ldots$. In this expansion $\delta^{g}$ lowers the gauge fixed antifield number by 1 and is related to the gauge fixed equations of motion including the fields of the non-minimal sector. The operator $\gamma^{g}$ depends on the choice of gauge fixing and is of gauge fixed antifield number 0 . Its action on the fields, after putting to zero the antifields, is often referred to as the BRST symmetry, because it corresponds to a rigid, global symmetry of the gauge fixed action. For generic gauge theories, this symmetry is only nilpotent on-the-gauge-fixedshell. Finally, the operator $\lambda^{g}$ is of gauge fixed antifield number 1, and the dots indicate operators of higher gauge fixed antifield number.

The cohomology classes corresponding to the operator $\gamma^{g}$ naturally arise in an operator formalism like the one we discuss in section 6. We discuss the connection between the gauge fixed and gauge invariant cohomology in detail in the next section. 


\section{Gauge Invariant and Gauge Fixed Cohomology}

Consider a solution $S\left[\phi, \phi^{*}\right]$ of the master equation. The gauge invariant cohomology is given by solutions of $\left(S\left[\phi, \phi^{*}\right], A\left[\phi, \phi^{*}\right]\right)=0$ up to solutions of the form $A=(S, B)$, where $A$ and $B$ are local functionals of the fields and antifields. The equivalence classes $[A]$ are independent of the variables of the non-minimal sector $B, \bar{C}$ and their antifields, and there is no reference to any gauge fixing. Furthermore, the gauge invariant classes $[A]$ can be described in terms of antifield-independent quantities: they are completely determined by classes $\left[A_{0}\right]$ satisfying $\gamma A_{0} \approx 0$, where trivial solutions are given by $A_{0} \approx \gamma B_{0}$.

The consistency condition that arises in standard approaches to renormalization is $\left(\tilde{S}\left[\phi, \tilde{\phi}^{*}\right], \tilde{A}\left[\phi, \tilde{\phi}^{*}\right]\right)=0$ with $\tilde{A}$ of ghost number 1 for the anomalies and of ghost number 0 for the counterterms, where $\tilde{S}\left[\phi, \tilde{\phi}^{*}\right]=S\left(\phi, \tilde{\phi}^{*}+\frac{\delta \Psi}{\delta \phi}\right)$ is the gauge fixed action where antifields are present. Here and in what follows tilded quantities refer to the gauge fixed theory. Solutions of the form $\tilde{A}=(\tilde{S}, \tilde{B})$ are trivial in the following sense: for $g=1$, the anomaly $\tilde{A}$ can be absorbed by adding to the Lagrangian the BRST breaking counterterm $-\tilde{B}$ and, for $g=0$, the counterterm $\tilde{A}$ can be absorbed by a canonical field/antifield redefinition. Because the presence of the antifields allows an implementation of the gauge-fixing as a (canonical) change of variables and because the non-minimal sector is cohomologically trivial, it can be shown that the equivalence classes of solutions $[\tilde{A}]$ are isomorphic to solutions $[A]$ of the gauge invariant cohomology.

The gauge fixed cohomology groups for any ghost number have been worked out in appendix A of [10] (see also [15]). It is shown that the BRST cohomology in $\mathcal{F}$ at ghost number $g$ is isomorphic to the direct sum of two spaces:

- The first space is a linear subspace of $H^{g}\left(\gamma^{g}, \mathcal{F}_{W}^{g}\right)$, where $\mathcal{F}_{W}^{g}$ is the space of onshell functionals (for the gauge fixed field equations). Each element of $H^{g}\left(\gamma^{g}, \mathcal{F}_{W}^{g}\right)$ determines a first order deformation of the gauge fixed action and the gauge fixed BRST symmetry and the subspace is determined by requiring that the deformed BRST symmetry is nilpotent (on the gauge fixed shell). An example of an element of $H^{g}\left(\gamma^{g}, \mathcal{F}_{W}^{g}\right)$ that does not fulfill this condition, and thus does not correspond to a local BRST cohomology class in $\mathcal{F}_{W}^{g}$ is the Curci-Ferrari mass term [16, 17].

- The second space completing $H^{g}(s, \mathcal{F})$ is isomorphic to the space of local BRST cohomology classes in $\mathcal{F}$, that, when expressed in terms of the gauge fixed variables and after putting the antifields to zero, become $\gamma^{g}$ exact on the gauge fixed shell. This space can also be described in terms of conserved currents at ghost number $g+1$ as we discuss below.

Our starting point is (A.3) of [10], which we explicitly spell out below. Recall that 
$\tilde{s}=\delta^{g}+\gamma^{g}+\lambda^{g}+\ldots$ is the gauge fixed BRST differential. The nilpotency of $\tilde{s}$ implies

$$
\left(\delta^{g}\right)^{2}=0, \quad \delta^{g} \gamma^{g}+\gamma^{g} \delta^{g}=0, \quad \delta^{g} \lambda^{g}+\lambda^{g} \delta^{g}+\left(\gamma^{g}\right)^{2}=0 .
$$

The cocycle condition for the first subspace is given by

$$
\begin{array}{r}
\gamma^{g} \tilde{A}_{0}+\delta^{g} \tilde{A}_{1}=0, \\
\lambda^{g} \tilde{A}_{0}+\gamma^{g} \tilde{A}_{1}+\delta^{g} \tilde{A}_{2}=0,
\end{array}
$$

while the coboundary condition is

$$
\tilde{A}_{0}=\gamma^{g} \tilde{B}_{0}+\delta^{g} \tilde{B}_{1} .
$$

Quantities that are denoted by a capital letter always refer to integrated quantities. Their subscripts here and in what follows denote the (gauge fixed) antifield number. Eqs. (4.8) and (4.10) show that $\left[\tilde{A}_{0}\right]$ is an element of $H\left(\gamma^{g}, \mathcal{F}_{W}^{g}\right)$. It was shown in detail in [10] that the condition (4.9) is equivalent to the nilpotency of a deformed BRST charge.

Let $j_{0}^{\mu}$ be the BRST current that generates the transformations $\gamma^{g} \phi^{A}$. Let also $\tilde{A}_{0}=$ $\int d^{n} x \mathcal{L}_{1}$ (the reason for the notation will be become clear in a moment) and $\tilde{A}_{1}=$ $-\int d^{n} x \Delta \phi^{A} \tilde{\phi}_{A}^{*}$. The un-integrated on-shell version of (4.8) and (4.9) is

$$
\begin{array}{r}
\gamma^{g} \phi^{A} \frac{\delta^{L} \mathcal{L}_{1}}{\delta \phi^{A}} \approx^{g} \partial_{\mu} j_{1}^{\mu}, \\
J^{(0) \mu A}\left(\frac{\delta^{L} \mathcal{L}_{1}}{\delta \phi^{A}}\right)+\Delta j_{0}^{\mu}+\gamma^{g} j_{1}^{\mu} \approx^{g} \partial_{\nu} T^{[\nu \mu]},
\end{array}
$$

where $J^{(0) \mu A}(\cdot)$ may involve spacetime derivatives acting on the quantity inside the parenthesis. The coboundary condition becomes

$$
\mathcal{L}_{1} \approx^{g} \gamma^{g} b_{0}+\partial_{\mu} k_{0}^{\mu}
$$

Eq. (4.11) follows straightforwardly from (4.8) by noting that $\delta^{g}$ acting on antifields yields the (gauge fixed) field equations. Eq. (4.11) implies that $\left(j_{0}^{\mu}+e j_{1}^{\mu}\right)$ is a conserved current of the theory with Lagrangian $\mathcal{L}_{0}+e \mathcal{L}_{1}$, where $\tilde{S}\left(\phi, \phi^{*}=0\right)=\int d^{n} x \mathcal{L}_{0}$. Let $\left(\gamma^{g}+e \Delta\right) \phi^{A}$ be the transformation rules generated by the modified BRST current. Relation (4.12), or equivalently (4.9), expresses the fact that the modified BRST charge is nilpotent, $\left(\gamma^{g}+e \Delta\right)^{2} \approx^{\prime} O\left(e^{2}\right)$ (see [10] for the details). Eq. (4.13) says that trivial solutions $\mathcal{L}_{1}$ are the ones that are weakly BRST exact, up to a total divergence. That $\mathcal{L}_{1}$ of the form of (4.13) indeed satisfies (4.11) and (4.12) is most easily seen on the equivalent formulations (4.8) and (4.9). Furthermore, by a (somewhat involved) computation one can show that (4.12) is equivalent to

$$
\vec{k}^{A \mu}\left(\frac{\delta^{L} \mathcal{L}_{1}}{\delta \phi^{A}}\right)+2 \gamma^{g} j_{1}^{\mu} \approx^{g} \partial_{\nu} T^{\prime[\nu \mu]}
$$


where $\vec{k}^{A \mu}$ may contain derivatives with respect to the fields and their derivatives, i.e. terms of the form $k^{A B \mu} \frac{\partial}{\partial \phi^{B}}\left(\frac{\delta^{L} \mathcal{L}_{1}}{\delta \phi^{A}}\right)$ etc.

The cocycle condition for the second subspace reads

$$
\begin{array}{r}
\delta^{g} \tilde{C}_{1}=0, \\
\gamma^{g} \tilde{C}_{1}+\delta^{g} \tilde{C}_{2}=0 .
\end{array}
$$

In un-integrated form, these read

$$
\begin{array}{r}
\partial_{\mu} \bar{l}_{0}^{\mu} \approx^{g} 0 \\
\gamma^{g} \bar{l}_{0}^{\mu} \approx^{g} \partial_{\nu} S^{[\nu \mu]} .
\end{array}
$$

Eq. (4.17) is a straightforward rewriting of (4.15), while (4.18) is derived by a standard application of descent equation techniques: we apply $\delta^{g}$ on (4.16) and use (4.7) and (4.15) and the fact that $d$ is acyclic. Notice that these conditions imply that $\bar{l}_{0}^{\mu}$ is a BRST invariant conserved current. Furthermore, given a particular solution $\left(\mathcal{L}_{1}, j_{1}^{\mu}\right)$ of equations (4.11) and (4.14), the remaining ambiguity in $j_{1}^{\mu}$ is precisely the general solution $\bar{l}_{0}^{\mu}$ of (4.17) and (4.18), i.e., $\left(\mathcal{L}_{1}, j_{1}^{\mu}+\bar{l}_{0}^{\mu}\right)$ satisfies (4.11) and (4.14) iff $\bar{l}_{0}^{\mu}$ solves (4.17) and (4.18).

The coboundary condition is

$$
\begin{array}{r}
0=\gamma^{g} \tilde{D}_{0}+\delta^{g} \tilde{D}_{1}, \\
\tilde{C}_{1}=\lambda^{g} \tilde{D}_{0}+\gamma^{g} \tilde{D}_{1}+\delta^{g} \tilde{D}_{2},
\end{array}
$$

which, in un-integrated form, reads

$$
\begin{array}{r}
\partial_{\mu} \rho_{0}^{\mu} \approx^{g}-\gamma^{g} \tilde{d}_{0}, \\
\bar{l}_{0}^{\mu} \approx^{g}-\gamma^{g} \rho_{0}^{\mu}+\partial_{\nu} R^{[\nu \mu]} .
\end{array}
$$

The derivation of these equations is similar to the ones in (4.17) and (4.18) (to derive (4.22) act on (4.19) by $\gamma^{g}$ and on (4.20) by $\delta^{g}$ and use (4.7)).

In summary, the cocycle condition of the gauge fixed cohomology,

$$
(\tilde{S}, \tilde{A})=0, \quad \tilde{A}=\tilde{A}_{0}+\left(\tilde{A}_{1}+\tilde{C}_{1}\right)+\left(\tilde{A}_{2}+\tilde{C}_{2}\right)+\ldots
$$

is equivalent to (4.8), (4.9), (4.15), (4.16), or in un-integrated form to (4.11), (4.12), (4.17), (4.18), and the coboundary condition

$$
\tilde{A}=(\tilde{S}, \tilde{B}), \quad \tilde{B}=\left(\tilde{B}_{0}+\tilde{D}_{0}\right)+\left(\tilde{B}_{1}+\tilde{D}_{1}\right)+\ldots
$$

is equivalent to (4.10), (4.19), (4.20), or in un-integrated form to (4.13), (4.21), (4.22). We thus see that $[\tilde{A}]$ is equivalent to $\left(\left[\tilde{A}_{0}\right],\left[\tilde{C}_{1}\right]\right)$, respectively to $\left(\left[\mathcal{L}_{1}\right],\left[\bar{l}_{0}^{\mu}\right]\right)$. This should be contrasted with the case of the gauge invariant cohomology, where the antifield independent part $\left[A_{0}\right]$ was sufficient to completely determine $[A]$. 


\section{An Example}

In this section we illustrate with a simple example the discussion in the previous section. In particular we demonstrate the emergence of non-trivial currents in specific gauges. This example was also briefly discussed in [10]. We consider Maxwell theory with a free massive scalar $\Phi$. The gauge invariant action is

$$
S_{0}=\int d^{4} x\left(-\frac{1}{4} F^{\mu \nu} F_{\mu \nu}-\frac{1}{2} \partial_{\mu} \Phi \partial^{\mu} \Phi-\frac{1}{2} m^{2} \Phi^{2}\right)
$$

The gauge invariant non-minimal solution of the master equation is

$$
S\left(\phi, \phi^{*}\right)=S_{0}+\int d^{4} x\left(A^{* \mu} \partial_{\mu} C+\bar{C}^{*} B\right)
$$

where $C$ is the ghost field, $\bar{C}$ is the antighost field and $B$ is the BRST auxiliary field. In the gauge invariant theory, $\int d^{4} x \Phi$ is BRST closed, $\left(S, \int d^{4} x \Phi\right)=0$, but not BRST exact, so $\left[\int d^{4} x \Phi\right]$ is a non-trivial class of $H^{0, n}(s \mid d)$.

We now consider fixing the gauge using the gauge fixing condition $\partial^{\mu} A_{\mu}=\mu \Phi$, where $\mu$ is a mass parameter. The limit $\mu \rightarrow 0$ yields the well-known Lorentz gauge. The gauge fixing fermion that implements this gauge is given by

$$
\Psi=\int d^{4} x \bar{C}\left(\frac{1}{2} \alpha B+\partial_{\mu} A^{\mu}+\mu \Phi\right)
$$

The gauge fixed action $S^{g}=S\left(\phi, \tilde{\phi}^{*}+\delta \Psi / \delta \phi\right)$ is equal to

$$
\begin{aligned}
S^{g}=\int d^{4} x(- & \frac{1}{4} F^{\mu \nu} F_{\mu \nu}-\frac{1}{2} \partial_{\mu} \Phi \partial^{\mu} \Phi-\frac{1}{2} m^{2} \Phi^{2}-\partial^{\mu} \bar{C} \partial_{\mu} C+\left(\frac{1}{2} \alpha B+\partial_{\mu} A^{\mu}+\mu \Phi\right) B \\
& \left.+\tilde{A}^{* \mu} \partial_{\mu} C+\tilde{\bar{C}}^{*} B\right) .
\end{aligned}
$$

We now set the antifields to zero. As is well known, the BRST variation of the antighost yields the gauge fixing condition,

$$
\gamma^{g} \bar{C} \approx^{g}-\frac{1}{\alpha}\left(\partial^{\mu} A_{\mu}+\mu \Phi\right)
$$

where the field equation of $B$ was used. Integrating we get

$$
\mu \int d^{4} x \Phi \approx^{g} \gamma^{g} \int d^{4} x \alpha \bar{C}
$$

The action (5.28) is invariant under constant shifts of the antighost, $\delta \bar{C}=\epsilon$. The corresponding Noether current is given by

$$
\bar{l}_{0}^{\mu}=\partial^{\mu} C=\gamma^{g} A^{\mu}
$$

This current is BRST invariant,

$$
\gamma^{g} \bar{l}_{0}^{\mu}=0
$$


Let us first consider the case $\mu=0$, i.e. the standard Lorentz gauge. In this case, the current $\bar{l}_{0}^{\mu}$ is trivial since the condition in (4.22) are satisfied with $\rho_{0}^{\mu}=-A^{\mu}$ and $\tilde{d}_{0}=-\bar{C}$. Furthermore, $\int d^{4} x \Phi$ is non-trivial, as in the gauge invariant formulation (the relation (5.30) just says that $\int d^{4} x \bar{C}$ is BRST closed).

We now discuss the case $\mu \neq 0$. The relation (5.30) implies that $\int d^{4} x \Phi$ is trivial. The current $\bar{l}_{0}^{\mu}$, however, is no longer trivial because the first eq. in (4.22) is not satisfied due to the last term in (5.29). We thus see that in this gauge we lost one non-trivial class at the level of $\mathcal{L}_{1}$ but gained another one at the level of $j_{1}^{\mu}$.

There is an elegant reformulation of this discussion when antifields are present. As discussed, $\int d^{4} x \Phi$ represents a non-trivial class in the gauge invariant formulation. Furthermore, the functional $\int d^{4} x \bar{C}^{*}$ has ghost number zero, is BRST closed, $\left(S, \int d^{4} x \bar{C}^{*}\right)=0$, but it is also BRST exact, $\int d^{4} x \bar{C}^{*}=\left(S, \int d^{4} x B^{*}\right)$, and thus it represents the trivial class. Notice that $\int d^{4} x \bar{C}^{*}$ generates (by the antibracket) arbitrary constant shifts of the antighost $\bar{C}$. This a trivial symmetry because it comes from a gauge symmetry: the action (5.26) is invariant under local shifts of the antighost.

Let us now consider the gauge fixed case. A simple computation yields

$$
\mu \int d^{4} x \Phi=\left(S^{g}, \int d^{4} x\left(\tilde{B}^{*}+\alpha \bar{C}\right)\right)-\int d^{4} x \tilde{\bar{C}}^{*} .
$$

We thus see that when $\mu=0, \int d^{4} x \tilde{\bar{C}}^{*}$ is trivial as in the gauge invariant formulation, but when $\mu \neq 0$ both $\int d^{4} x \Phi$ and $\int d^{4} x \tilde{\bar{C}}^{*}$ represent the same cohomology class.

We would like to emphasize that the discussion so far was about possible counterterms in the quantum effective action (and, as we discuss in the last section, about the local normalization ambiguity in the BSEG approach). There is a separate issue concerning quantum operators and whether they are trivial inside correlation functions, as we now discuss. To analyze this question we examine integrated correlation functions of $d$-exact operators, $\partial_{\mu} K^{\mu}$, with an arbitrary number of gauge invariant operators $O_{i}$, $\int d^{4} x\left\langle T\left[\left(\partial_{\mu} K^{\mu}\right)(x) O_{1}\left(x_{1}\right) \cdots O_{n}\left(x_{n}\right)\right]\right\rangle$. Of course, for the question to be well-posed the correlators should be free of IR divergences. Let us consider for concreteness the case of 2-point functions. Standard manipulations lead to

$$
\int d^{4} x\left\langle T\left[\left(\partial_{\mu} K^{\mu}\right)(x) O_{1}(y)\right]\right\rangle=-\int d^{3} x\left\langle\left[K^{0}(\vec{x}, t), O_{1}(\vec{y}, t)\right]\right\rangle+\int d^{4} x \partial_{\mu}^{x}\left\langle T\left[K^{\mu}(x) O_{1}(y)\right]\right\rangle
$$

The first term is standard, but the second term is usually dropped because it is the integral of a total derivative and it is usually assumed that all fields fall off sufficiently fast at infinity so that such terms can be ignored. It is such terms that are the focus of our analysis here.

Let us now consider $K^{\mu}=A^{\mu}$ and $O_{1}=\Phi$. In this case it is straighforward to compute explicitly all quantities. Because of the gauge-fixing there is an off-diagonal 
propagator,

$$
\left\langle T\left[A^{\mu}(x) \Phi(y)\right]\right\rangle=\mu \int \frac{d^{4} p}{(2 \pi)^{4}} e^{i p \cdot(x-y)} \frac{p^{\mu}}{p^{2}\left(p^{2}+m^{2}\right)} .
$$

It follows that

$$
\int d^{4} x \partial_{\mu}^{x}\left\langle T\left[A^{\mu}(x) O_{1}(y)\right]\right\rangle=i \frac{\mu}{m^{2}} .
$$

We thus find that in this specific example a correlator that is naively zero because it is the integral of a total derivative is actually non-zero.

Let us try to understand this result. Standard arguments imply that when an operator is BRST exact its correlation functions with any gauge invariant operators $O_{i}$ vanishes,

$$
\left\langle T\left[\left(\gamma^{g} A\right)(x) O_{1}\left(x_{1}\right) \cdots O_{n}\left(x_{n}\right)\right]\right\rangle=0
$$

where $A$ is any local operator. We can use this Ward identity with $A=\bar{C}$ to show (the well-known fact) that all correlation functions of the gauge fixing relation with gauge invariant operators vanish. In our case this implies,

$$
\mu\left\langle T\left[\Phi(x) O_{1}\left(x_{1}\right) \cdots O_{n}\left(x_{n}\right)\right]\right\rangle=-\left\langle T\left[\left(\partial_{\mu} A^{\mu}\right)(x) O_{1}\left(x_{1}\right) \cdots O_{n}\left(x_{n}\right)\right]\right\rangle .
$$

This relation tells us that the correlation functions of the gauge variant operator $\partial_{\mu} A^{\mu}$ are determined in this gauge by the correlation functions of the gauge invariant operator $\Phi$. Notice that the correlation functions $\left\langle T\left[\Phi(x) O_{1}\left(x_{1}\right) \cdots O_{n}\left(x_{n}\right)\right]\right\rangle$ are the same in all gauges (since they involve gauge invariant operators) but the correlation functions of $\left\langle T\left[\partial_{\mu} A^{\mu}(x) O_{1}\left(x_{1}\right) \cdots O_{n}\left(x_{n}\right)\right]\right\rangle$ depend on the gauge under consideration.

We now consider an integrated version of (5.38) with $O_{1}=\Phi$ and no other operators,

$$
\mu \int d^{4} x\langle T[\Phi(x) \Phi(y)]\rangle=-\int d^{4} x\left\langle T\left[\left(\partial_{\mu} A^{\mu}\right)(x) \Phi(y)\right]\right\rangle .
$$

First, the equal-time commutator between $A^{0}$ and $\Phi$ is zero (as an explicit computation shows) so one can freely move the derivative outside the time-ordering in the right hand side. The 1.h.s. is equal to

$$
\mu \int d^{4} x\langle T[\Phi(x) \Phi(y)]\rangle=-i \mu \int d^{4} x \frac{d^{4} p}{(2 \pi)^{4}} \frac{e^{i p \cdot(x-y)}}{p^{2}+m^{2}}=-i \frac{\mu}{m^{2}}
$$

so (5.39) yields (5.36).

As mentioned above, ordinarily integrals of total derivatives of correlators are set to zero because all fields are assumed to fall off sufficiently fast at infinity. The asymptotic behavior of $A_{\mu}$, however, depends on the gauge. In the current case the gauge fixing condition implies,

$$
\int_{\partial M} d \sigma^{n} A_{n}=-\mu \int_{M} d^{4} x \Phi
$$

which is non-zero. This (classical) statement is implemented in the quantum theory via the Ward identity (5.38). To take into account such non-trivial boundary conditions 
one may consider the cohomology of $d$ with compact support, i.e. a $d$-closed element is considered trivial if it is $d$-exact and vanishes at infinity. The operator $\partial_{\mu} A^{\mu}$ in thus non-trivial in $H_{\text {compact }}(d)$.

The existence of currents of the form of the second eq. in (4.22) that are non-trivial because the first condition in (4.22) is not satisfied always implies that there are such non-trivial $d$-closed operators. Indeed, the conservation of $\bar{l}_{0}^{\mu}$ in the second condition in (4.22) implies only that

$$
\gamma^{g} r \approx^{g} 0
$$

where $\partial_{\mu} \rho_{0}^{\mu} \approx^{g} r$. Now consider the correlation function of $\partial_{\mu} \rho_{0}^{\mu}$ with arbitrary number of gauge invariant operators. Naively, this should be equal to zero but because of (5.42) we obtain

$$
\int d^{4} x\left\langle T\left[\left(\partial_{\mu} \rho_{0}^{\mu}\right)(x) O_{1}\left(x_{1}\right) \cdots O_{n}\left(x_{n}\right)\right]\right\rangle=\int d^{4} x\left\langle T\left[r(x) O_{1}\left(x_{1}\right) \cdots O_{n}\left(x_{n}\right)\right]\right\rangle,
$$

which is (generically) non-zero since the right hand side is a correlation function of gauge invariant operators. In contrast, when the current is trivial, $r$ is $\gamma^{g}$-exact and the correlation function on the right hand side vanishes.

These considerations indicate that at the level of operators there is an alternative cohomology analysis to the one presented here and in the appendix of [10] where the non-trivial currents are due to $d$ having a non-trivial cohomology.

\section{Quantum Noether Method}

As already mentioned in the introduction, a construction of theories with global (=rigid) and/or local symmetries within the Bogoliubov-Shirkov-Epstein-Glaser (BSEG) operator approach was presented in [2, 3]. Recall that in this approach the $S$-matrix is constructed perturbatively in the asymptotic Fock space as a formal power series

$$
S(g)=1+\sum_{n=1}^{\infty} \frac{1}{n !} \int d x_{1}^{4} \cdots d x_{n}^{4} T_{n}\left(x_{1}, \cdots, x_{n} ; \hbar\right) g\left(x_{1}\right) \cdots g\left(x_{n}\right) .
$$

The coupling constant $g$ is replaced by a tempered test function $g(x) \in \mathcal{S}$ (i.e. a smooth function rapidly decreasing at infinity), which switches on the interaction. The $n$-point operator-valued distributions $T_{n} \in \mathcal{S}^{\prime}$ are the central objects, where $\mathcal{S}^{\prime}$ denotes the space of functionals on $\mathcal{S}$. They should be viewed as mathematically well-defined (renormalized) time-ordered products,

$$
T_{n}\left(x_{1}, \cdots, x_{n} ; \hbar\right)=T\left[T_{1}\left(x_{1}\right) \cdots T_{1}\left(x_{n}\right)\right]
$$

of a given specific coupling $T_{1}$, which is regarded as part of the definition of the theory (for example $T_{1}=i / \hbar: \phi^{4}:$ ). We note that the expansion in (6.1) is not a loop expansion. 
Each $T_{n}$ in (6.1) can receive tree-graph and loop-contributions. One can distinguish the various contributions from the power of $\hbar$ that multiplies them.

We are interested in constructing theories where the $S$-matrix is invariant under a certain symmetry operation, generated by a well-defined operator $Q$ in the asymptotic Fock space:

$$
[Q, S]=0 \text {. }
$$

The operator $Q$ acting on asymptotic fields generates their asymptotic transformation rules

$$
\left[Q, \phi^{A}\right\}=-i \hbar \gamma_{0} \phi^{A}
$$

where $[A, B\}$ denotes a graded commutator. The latter are necessarily linear in the asymptotic fields.

We would like to carry out the construction before the so-called adiabatic limit. Thus, instead of working with (6.3), we require that

$$
\left[Q, T_{n}\left(x_{1}, \ldots, x_{n} ; \hbar\right)\right\}=\sum_{l=1}^{n} \frac{\partial}{\partial x_{l}^{\mu}} T_{n / l}^{\mu}\left(x_{1}, \ldots, x_{n} ; \hbar\right)
$$

holds in the distributional sense for $n \geq 1$ and for some $T_{n / l}^{\mu}$; we use the abbreviation $\partial / \partial x_{l}^{\mu}=\partial_{\mu}^{l}$. For $n=1$, Eq. (6.5) reads

$$
\left[Q, T_{1}\right\}=\partial_{\mu} T_{1 / 1}^{\mu}
$$

and imposes restrictions on the starting point of the BSEG procedure, namely on the coupling $T_{1}{ }^{6}$ Once the coupling $T_{1}$ has been determined, the rest of Eqs. (6.5) impose relations between the constants left unspecified by the requirement of causality and Poincaré invariance. This is analogous to the situation in the conventional Lagrangian approach, where symmetry considerations restrict the possible terms in the Lagrangian and then the corresponding symmetries at the quantum level impose certain relations between the $Z$ factors.

Since different non-linear transformations may have the same linear limit, it is not $a$ priori obvious whether a theory constructed by BSEG satisfying (6.3) has any underlying non-linear structure at all. To address this issue one can work out the precise consequences of the operator equation (6.5) in specific models and try to reproduce the Ward identities derived in the Lagrangian approach, using the full non-linear transformation. ${ }^{7}$ An alternative and complementary approach is to try to find a direct correspondence

\footnotetext{
${ }^{6}$ The distributions $T_{n / l}^{\mu}$ in (6.5) are then given by the $T$-products of $(n-1)$ vertices $T_{1}$ and one vertex $T_{1 / 1}$ at the $l$ th position: $T\left[T_{1}\left(x_{1}\right) \cdots T_{1 / 1}^{\mu}\left(x_{l}\right) \cdots T_{1}\left(x_{n}\right)\right]$.

${ }^{7}$ This approach was followed in [18, 19] for the case of $S U(n)$ gauge theory in the Feynman gauge coupled to fermions where it was shown that (6.5) implies the Slavnov-Taylor identities for connected Green functions.
} 
between the Lagrangian approach and the BSEG formalism. The obvious advantage of such an approach is that it will provide a model-independent connection between the two approaches.

In order to establish such a connection to the Lagrangian approach, the symmetry constraint (6.5) was reformulated into an equivalent, but more transparent formulation based on the Noether method in [2]. Recall that in the Lagrangian approach Ward identities are derived using the conservation of symmetry currents inside correlation functions. The proposal in [2, 3] is to adopt this approach in the operator formalism: we constrain the local ambiguity by requiring that the corresponding Noether current be conserved inside correlation functions.

We start by including in the theory the coupling $g_{\mu} j_{0}^{\mu}$, where $j_{0}^{\mu}$ is the Noether current that generates the asymptotic (linear) symmetry transformations of the fundamental fields. Given such a current, one can obtain a corresponding interacting field operator $j_{0, i n t}^{\mu} \cdot{ }^{8}$ We then propose, as a general Ward identity [3]:

$$
\partial_{\mu} j_{0, i n t}^{\mu}=\partial_{\mu} g \tilde{j}_{1, i n t}^{\mu}
$$

where $\tilde{j}_{1, \text { int }}^{\mu}$ is another interacting current whose explicit form can be found in [3] but it will not be needed here. One of the main results of [3] is that the interacting current at tree level is exactly equal to the Noether current of the Lagrangian (non-linear) theory. It follows that in the naive adiabatic limit, $\partial_{\mu} g=0$, (6.8) reduces to the standard Ward identities. The condition (6.8) is a formal Laurent series in $\hbar$ so this condition is actually a set of conditions.

Using the fact that the right-hand side of (6.8) is linear in derivatives, one may provide an alternative Ward identity, which does not contain any explicit reference to $g(x)$. The reformulation is based on the distributional identity $\left(\sum_{i=1}^{n} \partial_{i}\right) \delta\left(x_{1}-x_{2}\right) \delta\left(x_{2}-\right.$ $\left.x_{3}\right) \ldots \delta\left(x_{n-1}-x_{n}\right)=0$. It follows that if one considers a symmetrized insertion of $j_{0}^{\mu}$ (instead of (6.7) ) then the term on the right-hand of (the symmetrized version of) (6.8) becomes proportional to a total derivative, which can then be removed by modifying the local ambiguity in correlation functions with one current insertion. This version of the Ward identity was presented in [2]. By construction, the two Ward identities imply exactly the same conditions on all correlators with no current insertions, but those that

\footnotetext{
${ }^{8}$ The perturbation series for the interacting field operator $j_{0, \text { int }}^{\mu}$ of a free field operator $j_{0}^{\mu}$ is given by the advanced distributions of the corresponding expansion of the $S$-matrix:

$$
j_{0, \text { int }}^{\mu}(g, x)=j_{0}^{\mu}(x)+\sum_{n=1}^{\infty} \frac{1}{n !} \int d^{4} x_{1} \ldots d^{4} x_{n} A d_{n+1}\left[T_{1}\left(x_{1}\right) \ldots T_{1}\left(x_{n}\right) ; j_{0}^{\mu}(x)\right] g\left(x_{1}\right) \ldots g\left(x_{n}\right),
$$

where $A d_{n+1}$ denotes the advanced operator-valued distribution with $n$ vertices $T_{1}$ and one vertex $j_{0}^{\mu}(x)$ at the $(n+1)$ th position; $g\left(x_{k}\right)$ is a tempered test function, which, in the adiabatic limit, becomes the coupling constant of the theory.
} 
have current insertions differ by the local terms just discussed. The Ward identity (6.8) has the advantage that it involves the current $j_{0, i n t}^{\mu}$ that renormalizes to become exactly equal to the Noether current of the Lagrangian formulation (in the symmetrized Ward identity the renormalization of $j_{0}^{\mu}$ involves the same terms, but with different combinatorial coefficients). Furthermore, it is more straightforward to analyse the anomalies of (6.8) than that of the symmetrized condition.

It was shown in [2, 3] that any theory with global/local symmetry that can be viewed as deformation of a free theory can be constructed by using the symmetry condition (6.8) (or the corresponding symmetrized condition) and the free Noether current $j_{0}^{\mu}$ as a starting point. This class of theories includes all perturbative QFTs. In addition, the equivalence of any theory consistently constructed in the BSEG formalism with a Lagrangian theory was established. The following was shown there:

1. The sum of $T_{1}$ and the tree-level normalizations that arise from the requirement (6.8) coincides with the Lagrangian that is invariant under the non-linear transformations generated by $j_{0, \text { int }}^{\mu}$. This shows that the full non-linear structure is present in the theory.

2. The interacting current $j_{0, \text { int }}^{\mu}$ is renormalized by condition (6.8) in such a way that it is, at tree level, exactly equal to the Lagrangian Noether current.

3. The loop normalization ambiguity is fixed in the same way as at tree level, provided the anomaly consistency condition has only trivial solutions. This means that the theory is stable under quantum correction.

It was also shown that condition (6.8) is equivalent to condition (6.5). The latter guarantees the invariance of the $S$-matrix under the corresponding asymptotic symmetry.

The consequence of all this is that the only information one needs in order to construct a perturbative quantum field theory with a given global symmetry is a set of free fields linearly realizing this symmetry. Even the first term in the $S$-matrix, which is usually regarded as an input in the BSEG formalism, is now derived using the quantum Noether condition.

Because the BSEG construction leads to the most general quantum field theory that is compatible with causality and Poincaré symmetry, the quantum Noether condition allows a determination of all consistent quantum field theories with non-linear symmetries that are compatible with the asymptotic symmetry represented by $j_{0}^{\mu}$.

Further consistency requirements on the theory follow by considering multicurrent correlation functions [10]. In particular, the two-current equation is

$$
\partial_{\mu}^{x} T\left[j_{0}^{\mu}(x) j_{0}^{\nu}(y) T_{1}\left(x_{1}\right) \cdots T_{1}\left(x_{n}\right)\right]=\partial_{\mu} g \tilde{j}^{\mu \nu},
$$


for some $\tilde{j}^{\mu \nu}$. In the case of a BRST symmetry, this additional constraint implements the nilpotency of the BRST transformation in the quantum theory. More generally, the two-current equation implements the algebra of symmetries.

The proofs given in 2, 3, 10, will not be repeated here. We discuss in the appendix, however, the so-called off-shell procedure. This alternative route to fix the local ambiguities and local breaking terms within the on-shell formalism provides structural (model-independent) information on the latter. It is this additional structural information that allows us to establish a cohomological analysis of the local ambiguities and the local anomalies within the BSEG operator approach.

Recently a new Ward identity was proposed within the BSEG approach [20, 21]. The so-called master Ward identity (MWI) is presented as a universal renormalization condition that expresses the symmetries of the underlying classical theory. The MWI is obtained by computing the difference,

$$
\partial_{x_{1}}^{\mu} T\left[W_{1}\left(x_{1}\right), \ldots, W_{n}\left(x_{n}\right)\right]-T\left[\partial^{\mu} W_{1}\left(x_{1}\right), \ldots, W_{n}\left(x_{n}\right)\right],
$$

using an algebraic equivalent of the Wick expansion formula (called N3 in 20]) and then demanding that the result is preserved by renormalization $\left(W_{1}, \ldots, W_{n}\right.$ are polynomials in free fields). We would like to emphasize that (as is well-known) the Wick expansion formula is not the most general solution of the BSEG construction. Actually the authors of [20] already encountered problems in reconciling (6.10) with known symmetries of certain models under consideration. In particular, it was found that the interacting Noether current in the case of massless Yang-Mills is not compatible with the MWI and the normalization condition N3, see formulae (157-160). Furthermore, in the case of the non-abelian Higgs model, the distributions $T_{n}$ fulfilling the MWI have to be modified due to certain non-linear couplings introduced by the BRST symmetry. The resulting $n$-point distributions $T_{n}^{N}$ violate the MWI and N3 as stated below formula (215). Since the MWI does not capture the full non-linear structure of symmetries (as these examples show), it seems likely that the theory satisfying MWI will have more anomalies and not be stable under quantum corrections.

\section{Gauge Fixed Cohomology and the QNM}

In [2, 3] the constraints imposed by the quantum Noether condition were analysed to all orders. Let $\mathcal{L}_{n}$ denote the local normalization ambiguity of $T_{n}$

$$
T_{n}\left[T_{1}\left(x_{1}\right) \ldots T\left(x_{n}\right) ; \hbar\right]=T_{c, n}\left[T_{1}\left(x_{1}\right) \ldots T\left(x_{n}\right) ; \hbar\right]+n ! \frac{i}{\hbar} \mathcal{L}_{n}(\hbar) \delta\left(x_{1}, \ldots, x_{n}\right),
$$

where $T_{c, n}$ denotes a reference splitting solution. At tree level, this is defined by using the Feynman propagator in tree-graphs. The Lagrangian is then identified with the tree-level 
contribution to $\mathcal{L}=\mathcal{L}_{0}+e \mathcal{L}_{1}+\ldots$ [2]. Here $\mathcal{L}_{0}$ is the Lagrangian of the free theory, and $e$ is the coupling constant of the theory ${ }^{9}$. It was shown in [2, 3] that the condition imposed by (6.8) on the local terms $\mathcal{L}_{n}$ coincides with the equations obtained in the classical Noether method. These considerations, thus, lead to an BSEG construction of all theories which are associated at the classical level with a Lagrangian invariant under some (non-linear) symmetry.

A particular case of theories where the construction applies is that of gauge theories, the relevant global symmetry being the BRST symmetry. In this case one can obtain the most general local terms compatible with gauge invariance using the antifield formalism. The objective of [10] was to show that the set of solutions of the quantum Noether conditions coincides with the set of solutions given by the antifield method.

In this case, the one-current quantum Noether condition (6.8) implies

$$
\gamma^{g} \mathcal{L}_{1}=\partial_{\mu} \mathcal{L}_{1}^{\mu}-\Delta \phi^{A} \frac{\delta^{L} \mathcal{L}_{0}}{\delta \phi^{A}},
$$

whereas the two-current Noether condition (6.9) yields

$$
J_{0}^{\mu A}\left(\frac{\delta \mathcal{L}_{1}}{\delta \phi^{A}}\right)+\gamma^{g} j_{1}^{\mu}+\Delta j_{0}^{\mu}=\partial_{\nu} T_{1}^{[\nu \mu]}+J_{1}^{\mu A}\left(\frac{\delta^{L} \mathcal{L}_{0}}{\delta \phi^{A}}\right) .
$$

Eq. (7.12) defines $\Delta \phi^{A}$. It was shown in [3] that these transformations are generated by $j_{1}^{\mu}$, and that furthermore $j_{1}$ is related to $\mathcal{L}_{1}$ by

$$
j_{1}^{\mu}=-\mathcal{L}_{1}^{\mu}+\frac{\partial \mathcal{L}_{1}}{\partial\left(\partial_{\mu} \phi^{A}\right)} \gamma^{g} \phi^{A} .
$$

Because these equations coincide with (4.11) and (4.12), the most general solution $\left(\mathcal{L}_{1}, j_{1}^{\mu}\right)$ are given by the gauge fixed BRST cocycles discussed in section 4 . Thus, we see that the QNC correctly gives the cocycle conditions of the gauge fixed cohomology.

We now turn to the issue of the coboundary terms. First consider the terms of the form (4.13). Inserting them in (6.1) we see that they are indeed trivial: total derivatives can be ignored in the (naive) adiabatic limit, and the asymptotic fields satisfy their field equation. Furthermore, BRST exact terms vanish when acting on physical states (since the latter are BRST closed).

Let us now consider the coboundary condition (4.21) and (4.22),

$$
\begin{array}{r}
\partial_{\mu} \rho_{0}^{\mu}=-d^{A} \frac{\delta^{L} \mathcal{L}_{0}}{\delta \phi^{A}}-\gamma^{g} \tilde{d}_{0}, \\
\bar{l}_{0}^{\mu}=-\gamma^{g} \rho_{0}^{\mu}+d^{\mu A} \frac{\delta^{L} \mathcal{L}_{0}}{\delta \phi^{A}}+\partial_{\nu} R^{[\nu \mu]} .
\end{array}
$$

\footnotetext{
${ }^{9}$ We use the convention of keeping the coupling constant explicit, in which case the adiabatic limit is $g(x) \rightarrow 1$.
} 
Recall that the starting point in the BSEG procedure is a set of asymptotic fields and their commutation relations, or equivalently their propagators. From these data one may construct a gauge fixed Lagrangian $\mathcal{L}_{0}$ that yields these propagators. As we discussed, the gauge fixed Lagrangian and the gauge fixed BRST current are associated with a gauge invariant Lagrangian $L$ and a corresponding BRST current. Suppose now that we change variables and choose another gauge, such that the resulting gauge fixed action changes only by a total derivative. The propagators of the gauge fixed theory remain invariant under this combined operation, but the BRST current can change. Clearly, the theory constructed using either the original or the new BRST current is the same. In this sense the difference between the two BRST currents is a trivial current. In other words, currents that can be removed by a combined change of gauge and of variables are trivial. The current $\bar{l}_{0}^{\mu}$ satisfying (7.15) and (17.16) is such a case. Indeed, consider the change of variables and gauge

$$
\phi^{A} \rightarrow \phi^{A}+e d^{A}, \quad \Psi \rightarrow \Psi-e \tilde{d}_{0},
$$

where $\Psi$ is the gauge fixing fermion. The gauge fixed Lagrangian changes by

$$
\mathcal{L}_{0} \rightarrow \mathcal{L}_{0}+e d^{A} \frac{\delta^{L} \mathcal{L}_{0}}{\delta \phi^{A}}+e \gamma^{g} \tilde{d}_{0}=\mathcal{L}_{0}-e \partial_{\mu} \rho_{0}^{\mu},
$$

where we used (7.15). The change of variables and of gauge entails a change in the BRST current. The details are somewhat involved but the final answer is that the original current changes by a current $e \bar{l}_{0}^{\mu}$ of the form (17.15) and (7.16). This analysis tell us that one should include all non-trivial currents $\bar{l}_{0}^{\mu}$ in the Noether procedure.

\section{Acknowledgments}

TH would like to thank R. Nest, F. Scheck, and E.Vogt for the invitation and for the organization of a stimulating meeting between mathematicians and physicists. KS is supported by NWO. The work of GB is supported in part by the "Actions de Recherche Concertées" of the "Direction de la Recherche Scientifique-Communauté Française de Belgique, by a "Pôle d'Attraction Interuniversitaire" (Belgium), by IISN-Belgium (convention 4.4505.86), by Proyectos FONDECYT 1970151 and 7960001 (Chile) and by the European Commission RTN program HPRN-CT00131, in which the author is associated to K. U. Leuven.

\section{A Off-Shell Procedure}

The purpose of this appendix is to review the so-called off-shell procedure presented in [2]. In order to avoid any misunderstandings, it should be stressed that the (well- 
defined) off-shell formalism is nothing else than a shortcut to fix the local ambiguities and local breaking terms within the on-shell BSEG formalism ${ }^{10}$. The advantage of the off-shell procedure is that it provides structural (model-independent) information on the latter, which allows us to establish a cohomological analysis of the local normalization ambiguity.

The symmetrized quantum Noether condition $^{11}$ is given by [2]:

$$
\partial_{\mu} \mathcal{J}_{n}^{\mu}\left(x_{1}, \cdots, x_{n} ; \hbar\right)=\sum_{l=1}^{n} \partial_{\mu}^{l} \mathcal{J}_{n / l}^{\mu}=0
$$

where

$$
\mathcal{J}_{n / l}^{\mu}=T\left[T_{1}\left(x_{1}\right) \cdots j_{0}^{\mu}\left(x_{l}\right) \cdots T_{1}\left(x_{n}\right)\right]
$$

We use the abbreviation $\partial / \partial x_{l}^{\mu}=\partial_{\mu}^{l}$. For $n=1$ we have the condition $\mathcal{J}_{1}^{\mu}\left(x_{1}\right)=j_{0}^{\mu}\left(x_{1}\right)$.

We start the quantum Noether construction [2] by noting that having satisfied our fundamental quantum Noether condition (A.20) for all $m<n$, Eq. (A.20) at the $n$th order can be violated by a local distribution $A_{n}(\hbar)$ (which we shall call local breaking term) only:

$$
\partial_{\mu} \mathcal{J}_{n}^{\mu}\left(x_{1}, \cdots, x_{n} ; \hbar\right)=\sum_{l=1}^{n} \partial_{\mu}^{l} \mathcal{J}_{n / l}^{\mu}=A_{n}(\hbar)
$$

The conventional inductive BSEG construction may be applied to work out the consequences of (A.20) and to fix the local terms $A_{n}(\hbar)$ in (A.22). However, there is an alternative route which was worked out in great detail in [2], and this under the name "off-shell formulation of the inductive hypothesis". To understand how this off-shell formulation simplifies the calculation of local on-shell terms $A_{n}(\hbar)$ arising from tree-level contractions, the traditional way to do such a calculation should first be described in more detail: In

${ }^{10}$ The field operators of the BSEG construction fulfill the free field equations and in this sense the formalism is on-shell.

${ }^{11}$ As mentioned in section [6] this symmetrized condition is equivalent to the Ward identity (6.8), $\partial_{\mu} j_{0, \text { int }}^{\mu}=\partial_{\mu} g \tilde{j}_{1, \text { int }}^{\mu}$, in its implications on physical correlation functions with no current insertions (see 3] for an explicit proof). The Ward identity (6.8) can be written in terms of T-products as

$$
\left.\partial_{\mu}^{x} T_{n+1}\left[T_{1}\left(x_{1}\right) \ldots T_{1}\left(x_{n}\right) ; j_{0}^{\mu}(x)\right]=-\sum_{j=1}^{n} T_{n}\left[T_{1}\left(x_{1}\right) \ldots \widehat{T_{1}\left(x_{j}\right.}\right) \ldots T_{1}\left(x_{n}\right) ; \tilde{j}_{1}^{\mu}(x)\right] \partial_{\mu}^{x_{j}} \delta\left(x_{j}-x\right) .
$$

These distributions get smeared out by $g\left(x_{1}\right) \ldots g\left(x_{n}\right) \tilde{g}(x)$, where the test-function $\tilde{g}$ differs from $g$. One easily verifies that the symmetrization in all variables (smearing out A.19) by $g\left(x_{1}\right) \ldots g\left(x_{n}\right) g(x)$ ) just leads to the symmetrized quantum Noether condition (A.20). As is described in the main text, the two Ward identities lead to a different normalization of correlators with current insertions, but one can readily obtain the normalization needed to satisfy (A.20) from the normalization needed to satisfy (6.8) (and vice versa) using the fact that the former is a symmetrized version of the latter. The explicit local normalization terms at order $n$ of the one-current correlators are given explicitly in [3] for both Ward identities. 
order to obtain the local terms, one first constructs ${ }^{12} T_{c, n}\left[j_{0}^{\mu}\left(x_{1}\right) T_{1}\left(x_{2}\right) \ldots T_{1}\left(x_{n}\right)\right]$, differentiates with respect to the variable of the current and symmetrizes in all variables. $T_{c, n}\left[j_{0}^{\mu}\left(x_{1}\right) T_{1}\left(x_{2}\right) \ldots T_{1}\left(x_{n}\right)\right]$ involves many terms and there will be a large number of cancellations after differentiating and symmetrizing. In particular, one already knows from Eq. A.22 that all non-local terms will cancel one another. So the idea (which gets implemented with the help of the off-shell trick) is to concentrate on possible local terms, anticipating the cancellation of all non-local terms.

By induction hypothesis, one has for all $m<n$,

$$
\sum_{l=1}^{m} \partial_{\mu}^{l} \mathcal{J}_{m / l}^{\mu}=0
$$

However, the assumption that the quantum Noether method works successfully means that there exist local normalizations such that (A.20) is satisfied when the field equations are satisfied. Therefore one rewrites -just as a trick- the induction hypothesis (A.23) by relaxing the field equations of the fields $\phi^{A}$ (which will be denoted by $\mathcal{K}_{A B} \phi^{B}$ in the following):

$$
\sum_{l=1}^{m} \partial_{\mu}^{l} \mathcal{J}_{m / l}^{\mu}=* R^{A ; m}(\hbar) \mathcal{K}_{A B} \phi^{B} \delta^{(m)} *, \quad m<n .
$$

It should be stressed again that the off-shell terms on the right-hand side are understood just as a calculational device to fix the local on-shell terms $A_{n}(\hbar)$ out of tree contractions in the next inductive step $(n-1) \rightarrow n$. In the following, the stars * are always used within the exact equations of the BSEG construction, as in (A.24), in order to indicate these offshell terms. Note that all these terms are just zero within the BSEG on-shell formalism. Nevertheless, the off-shell terms have a well-defined meaning and can be constructed straightforwardly with the help of BSEG quantities. The uniqueness of these terms will be discussed below.

The crucial point of the construction is that exactly those terms that are proportional to the field equations are the only source of the local on-shell terms $A_{n}(\hbar)$ in the next step of the induction within tree-level contractions: at order $n$ the so-called relevant contractions, namely the contractions between the $\phi^{B}$ in the right-hand side of (A.24) and $\phi$ within another local term, lead to the local terms. This implies, in particular, that no local term arises from terms in the causal distribution at the order $n$ that are products of more than two $T$ products. This is in accordance with the diagrammatic picture of creation of local terms that was discussed in [2]. In this manner one gets the following general formula for the local on-shell term $A_{c, n}$ arising through tree-level contractions at level $n[2]$ :

$$
A_{c, n}(\text { tree })=\sum_{\pi \in \Pi^{n}} \sum_{m=1}^{n-1} \underbrace{\partial_{\mu} \mathcal{J}_{m}^{\mu}\left(x_{\pi(1)}, \ldots, x_{\pi(m)}\right) N_{n-m} \delta\left(x_{\pi(k+1)}, \ldots, x_{\pi(n)}\right)}_{\text {relevant contractions }}
$$

\footnotetext{
${ }^{12} T_{c}$ denotes the natural splitting solution, i.e. the Feynman propagator is used in tree-graphs.
} 
where it is understood that only relevant contractions are made on the right-hand side. The factors $N_{n-m}$ are tree-level normalization terms of the $T$-products ${ }^{13}$ solution that contain $(n-m) T_{1}$ vertices. In all equations, Wick-ordering is always understood.

Now we have to identify the field-equation terms $R^{A ; m}(\hbar) \mathcal{K}_{A B} \phi^{B} \delta^{(m)}$ in (A.24). Let us recall that, generically, after natural splitting (this refers to tree-level graphs, for loop graphs one uses some reference-splitting solution) we end up with

$$
\partial_{\mu} \mathcal{J}_{c, m}^{\mu}=A_{c, m}
$$

The assumption that the quantum Noether method works successfully means that the (on-shell) anomaly $A_{c, m}$ is a divergence up to terms $B_{m}$ which vanish when the free-field equations are used, i.e.

$$
A_{c, m}=\partial_{\mu} A_{c, m}^{\mu}+* B_{m} *
$$

where $A_{m, c}^{\mu}$ and $B_{m}$ are some local distributions (since $A_{c, m}$ is local). This decomposition is not unique since one can move derivatives of field-equation terms from $B_{m}$ to $A_{c, m}^{\mu}$. This freedom is fixed by demanding that $B_{m}$ contain no derivatives of field equations (for details see [2], section 4.2); thus, we can identify $B_{m}$ with the right-hand side of (A.24), while the divergence term $\partial_{\mu} A_{c, m}^{\mu}$ can be canceled by the introduction of an additional normalization term of the $T$-product $\mathcal{J}_{n / l}^{\mu}$.

An additional ambiguity is related to the additional global symmetries of the free action. If we make the transformation

$$
A_{c, m}^{\mu} \rightarrow A_{c, m}^{\mu}+\tilde{j^{\mu}} \delta^{m} ; \quad R^{A ; m} \rightarrow R^{A ; m}-\tilde{\gamma} \phi^{A}
$$

where $\tilde{j}^{\mu}$ is a Noether current that generates the additional symmetry transformations $\tilde{\gamma} \phi^{A}$, then the right-hand side of (A.27) remains unchanged. The meaning of this ambiguity is that different symmetries can mix with each other. In general, one must include in the construction all currents that have non-trivial commutation relations among themselves. In the case of BRST symmetry, we have seen in the main text that one should include all non-trivial global currents of ghost number 1 in the construction in order to correctly account for all cohomology.

Having identified the field-equation terms as the only source of local terms out of tree contractions and having clarified the ambiguities due to the field equation we can use the off-shell trick to analyse the quantum Noether condition - first at tree level. Let us define

$$
\gamma_{(m-1)} \phi^{A}=\frac{1}{m !} R^{A ; m}\left(\hbar^{0}\right) ; m>1 .
$$

\footnotetext{
${ }^{13}$ When one refers to local normalization terms in tree graph contributions in BSEG they are always defined with respect to the natural splitting (see footnote [12).
} 
We have explicitly shown in [2, 3] that,

$$
\gamma \phi^{A}=\sum_{m=0}^{k} g^{m} \gamma_{m} \phi^{A},
$$

are symmetry transformation rules that leave the Lagrangian invariant (up to total derivatives):

$$
\mathcal{L}=\sum_{m=0}^{k^{\prime}} g^{m} \mathcal{L}_{m},
$$

where $k$ and $k^{\prime}$ are integers (which may be infinity). The Lagrangian $\mathcal{L}$ is determined from the tree-level normalization conditions as follows,

$$
\mathcal{L}_{m}=\frac{\hbar}{i} \frac{N_{m}}{m !}, \quad \text { for } \quad m>1
$$

where $N_{m}$ denotes the local normalization ambiguity of $T_{m}\left[T_{1}\left(x_{1}\right) \ldots T_{1}\left(x_{m}\right)\right]$ in tree graphs defined with respect to the natural splitting solution. For $m=1, \mathcal{L}_{1}=(\hbar / i) T_{1}$. Note that we regard (A.32) as the definition of $\mathcal{L}_{m}$ within the BSEG approach. Details can be found in 2] where we also discuss the QNC at loop level.

\section{References}

[1] S. Deser, Self-interaction and gauge invariance, Gen. Rel. and Grav. I,1 (1970) 9.

[2] T. Hurth and K. Skenderis, Quantum Noether method, Nucl. Phys. B541 (1999) 566-614 hep-th/9803030.

[3] T. Hurth and K. Skenderis, The quantum Noether condition in terms of interacting fields, Lecture Notes Phys. 558 (2000) 86 hep-th/9811231.

[4] N.N. Bogoliubov and D.V. Shirkov, Introduction to the Theory of Quantized Fields (Interscience, New York, 1959).

[5] H. Epstein and V. Glaser, in: Statistical Mechanics and Quantum Field Theory, Proceedings of the 1970 Summer School of Les Houches, eds. C. de Witt and R. Stora, New York, Gordon and Breach, 1971; The role of locality in perturbation theory, Ann. Inst. Poincaré 29 (1973) 211.

[6] H. Epstein and V. Glaser, Adiabatic limit in perturbation theory, in G. Velo, A.S. Wightman (eds.), Renormalization Theory (D.Reidel Publishing Company, Dordrecht 1976), p.193; O. Piguet and A. Rouet, Symmetries in perturbative quantum field theory, Phys. Rep. 76 (1981) 1. 
[7] H. Epstein, V. Glaser and R. Stora, General properties of the $n$-point functions in local quantum field theory, in J. Bros, D. Jagolnitzer (eds.), Les Houches Proceedings 1975; G. Popineau and R. Stora, A pedagogical remark on the main theorem of perturbative renormalization theory, unpublished; R. Stora, Differential algebras, ETH lectures (1993), unpublished.

[8] G. Scharf, Finite Quantum Electrodynamics, The Causal Approach, Text and Monographs in Physics (Springer, Berlin 1995).

[9] C. Becchi, A. Rouet and R. Stora, "Renormalization of the abelian Higgs-Kibble model", Commun. Math. Phys. 42 (1975) 127 ; "Renormalization of gauge theories", Ann. Phys. (NY) 98 (1976) 287; I.V. Tyutin, "Gauge invariance in field theory and statistical mechanics", Lebedev preprint FIAN, n039 (1975); J. ZinnJustin, "Renormalisation of gauge theories" in Trends in elementary particle theory, Lecture notes in Physics $n^{0} 37$ (Springer, Berlin, 1975) ; Quantum Field Theory and Critical Phenomena, $3^{\text {rd }}$ (Edition Clarendon Press, Oxford, 1996); I.A. Batalin and G.A. Vilkovisky, Gauge algebra and quantization, Phys. Lett. 102B (1981) 27.

[10] G. Barnich, M. Henneaux, T. Hurth, and K. Skenderis, Cohomological analysis of gauge fixed gauge theories, Phys. Lett. B492 (2000) 376-384 hep-th/9910201.

[11] M. Henneaux and C. Teitelboim, Quantization of Gauge Systems, (Univ. Press, Princeton, 1992).

[12] J. Gomis, J. Paris and S. Samuel, Antibracket, antifields and gauge theory quantization, Phys. Rep. 259 (1995) 1 hep-th/9412228.

[13] O. Piguet and S. P. Sorella, Algebraic Renormalization, Lecture Notes in Physics (Springer, Berlin, 1995).

[14] G. Barnich, F. Brandt, and M. Henneaux, Local BRST cohomology in gauge theories, Phys. Rep. 338 (2000) 439 hep-th/0002245.

[15] M. Henneaux, "On the gauge-fixed BRST cohomology", Phys. Lett. B 367 (1996) 163, hep-th/9510116.

[16] G. Curci and R. Ferrari, On a class of Lagrangian models for massive and massless Yang-Mills fields, Nuov. Cim. 32A (1976) 151; 35A (1976) 1.

[17] J. de Boer, K. Skenderis, P. van Nieuwenhuizen and A. Waldron, On the renormalizability and unitarity of the Curci-Ferrari model for massive vector bosons, Phys. Lett. B367 (1996) 175 hep-th/9510167|; T. Hurth, Higgs-free massive nonabelian gauge theories, Helv. Phys. Acta 70 (1997) 406 hep-th/9511176; F. Brandt, "Deformations of global symmetries in the extended antifield formalism", J. Math. Phys. 40 (1999) 1023, hep-th/9804153. 
[18] M. Dütsch, T. Hurth and G. Scharf, Causal construction of Yang-Mills theories, Nuov. Cim. 106A (1993) 1029, 107A (1994) 375, 108A (1995) 679, 108A (1995) 737, Gauge invariance of massless QED, Phys. Lett. B327 (1994) 166 hep-ph/9403399.

[19] T. Hurth, Nonabelian gauge theories: the causal approach, Annals of Phys.(NY) 244 (1995) 340 hep-th/9411080; Nonabelian gauge symmetry in the causal EpsteinGlaser approach, Int. J. Mod. Phys. A12 (1997) 4461 hep-th/9511139.

[20] M. Dutsch and F. M. Boas, The master Ward identity, Rev. Math. Phys. 14 (2002) 977 hep-th/0111101.

[21] M. Dutsch and K. Fredenhagen, The master Ward identity and generalized Schwinger-Dyson equation in classical field theory, hep-th/0211242. 